\title{
EGG WHITE ALBUMIN FORM COMPLEX WITH ASPIRIN AND CAFFEINE AND ITS ROLE AS FREE RADICAL SCAVENGER
}

\author{
GATRA ERVI JAYANTI ${ }^{1}$, SRI WIDYARTI ${ }^{1}$, AKHMAD SABARUDIN ${ }^{2}$, SUTIMAN BAMBANG SUMITRO ${ }^{1 *}$ \\ ${ }^{1}$ Department of Biology, Faculty of Mathematics and Natural Sciences, Brawijaya University, Malang, 65145, Indonesia. ${ }^{2}$ Department of \\ Chemistry, Faculty of Mathematics and Natural Sciences, Brawijaya University, Malang 65145, Indonesia.
}

Email: sutiman@ub.ac.id

Received: 24 February 2018, Revised and Accepted: 14 April 2018

ABSTRACT

Objective: Egg white protein (ovalbumin) is well known to be freshly consumed in Indonesia as traditional medicine, or it is usually known as "Jamu." Ovalbumin, as well as egg white albumin, is able to form complex compounds with other substances through the formation of weak chemical and physical bonds. The objective of this study is to understand the behavior of ovalbumin as radical scavenger when it binds to antioxidants such as aspirin and caffeine (as a complex).

Methods: In this study, docking sites and ovalbumin as scavenger were studied using computer-modeling software. An ovalbumin was used only for computer modeling, whereas in the wet laboratory, the freeze-drying albumin was used for electron spin resonance (ESR) and Fourier transforminfrared (FTIR) spectroscopy to determine its ability as a scavenger and functional groups, respectively. Albumin solution was applied to measure the viscosity.

Results: The variability of tridimensional structures of aspirin was investigated after binding with ovalbumin. However, these structures cannot be seen clearly on caffeine. The root-mean-square deviation analysis showed that aspirin, as well as caffeine, altered the dynamic conformation of ovalbumin. The complex of aspirin-ovalbumin-caffeine which was treated at a temperature of $-70^{\circ} \mathrm{C}$ showed intermolecular force. ESR results showed that the complex compounds could effectively reduce more free radicals when compared to aspirin or caffeine. The existence of aromatic compounds (as confirmed by FTIR) was useful for the scavenger molecules and chemical interaction occurs. The viscosity of the complex was similar with normal gastric mucus, which associated with radical scavenger.

Conclusion: The characters of albumin when it binds to aspirin and caffeine indicated that scavenging activity of the complex and the viscosity showed an important result to be physiological scavengers of free radicals.

Keywords: Egg white albumin, Complex modeling, Free radical scavenger.

(c) 2018 The Authors. Published by Innovare Academic Sciences Pvt Ltd. This is an open access article under the CC BY license (http://creativecommons. org/licenses/by/4. 0/) DOI: http://dx.doi.org/10.22159/ajpcr.2018.v11i7.25440

\section{INTRODUCTION}

Fresh ovalbumin from avian eggs is usually used in Indonesia as traditional medicine, which is commonly known as "Jamu." Raw egg, which is mixed with coffee, is useful for the prevention of fatigue and body recovery. It is reported that ovalbumin has a scavenging activity. Ovalbumin peptides consist of Tyr-Ala-Glu-Glu-Arg-Tyr-Pro-Ile-Leu, which have strong angiotensin I-converting enzyme inhibitor and exhibited a high radical scavenging activity [1]. It is highly probable that the complex of caffeine and ovalbumin has strong free radical scavenging activity. The free radical scavenging activity may have beneficial application to reduce overwhelmingly production of free radicals. The rationale in this study is to improve affectivity of physiological oxidant scavenger system. A single antioxidant may turn into pro-oxidant in the presence of transition metal ions, resulting in accumulative free radical. During interaction with a single antioxidant, metal ions can be reduced, oxidized, and then rereduced (redox cycling) that can generate reactive oxygen species. Inversely, a complex antioxidant can scavenge free radical without accompanied by the formation of new radical species because of a lot of electron on its surface. We are reporting model study of interaction of ovalbumin and caffeine, as well as aspirin with the objective of studying the potential as oxidant scavenger.

Several biological activities of egg white were antioxidant properties, antimicrobial activities, antiadhesive properties, immunomodulatory, anticancer, and nutrient bioavailability [2]. Furthermore, ovalbumin, as well as egg white albumin, can produce complex compounds when they interact with other molecules. Weak chemical and physical bonds may be responsible for these interactions. It is known that ovalbumin is poorly digested and absorbed in a digestive tract, which has a potential to be used as a transporter. The rate of ovalbumin digestion is considerably slower than hydrolysis of bovine hemoglobin or globin, even by pepsin [3]. Ovalbumin can be digested thermally [4].

The heavy metal can be chelated and metal ion can be trapped within the sulfhydryl bonds of protein by ovalbumin. The chelating function prevents the absorption of the metals into a gastrointestinal cell and poisoning [5]. The capacity of ovalbumin antioxidant can be improved by the addition of aspirin and caffeine. It can form complexes with aspirin and caffeine as a complex of scavenger because it is easy to receive and to release the ligand [3]. The complex receives and releases electrons as it functions as a free radical scavenger.

Aspirin has an antioxidant effect [6]. In low dose, aspirin is effective in preventing heart attacks, strokes, and blood coagulation, and it is used to prevent certain type of cancer, particularly colorectal cancer [7]. Caffeine is a natural product from several plants and is derivative of a methylxanthine. It acts as a central nervous stimulant and resorting alertness. Furthermore, it can scavenge hydroxyl radicals by the "Fenton reaction" effectively $[8,9]$. Due to the valuable properties of molecules that have been mentioned before, our study focuses on the behavior of ovalbumin as a transporter and radical scavenger when it binds to antioxidants such as aspirin and caffeine. The use of ovalbumin is only for computer modeling, while egg white albumin is applied in the 
wet laboratory. Egg white albumin was utilized because it commonly used for "Jamu," easily obtained and inexpensive. From this related background, the combination of egg white albumin with aspirin and caffeine as a complex is expected to be more effective scavenger. To the best of our knowledge, there is no any report dealing with a complex antioxidant of aspirin-ovalbumin-caffeine. As a free radical scavenger, this complex is more effective than that of its counterparts (a single antioxidant). Accordingly, the objective of this study is to understand the behavior of ovalbumin as radical scavenger when it binds to antioxidants such as aspirin and caffeine (as a complex).

\section{METHODS}

In this study, computer modeling of ovalbumin, aspirin, and caffeine was provided from NCBI and PubChem database [10,11]. Docking sites and conformational properties of ovalbumin were observed using Pyrex [12] and Pymol [13]. LigPlot was used to visualize the chemical bond [14], and Yasara was used for root-mean-square deviation (RMSD) analysis [15]. Docking was proceeded by entering aspirin, caffeine, and ovalbumin molecules to Pyrex, and subsequently visualized by Pymol. Structural changes of the simulated molecules were observed using the RMSD analysis, i.e., ovalbumin, ovalbumin-aspirin, ovalbumincaffeine, and complex of aspirin-ovalbumin-caffeine. We would confirm in silico study using ovalbumin, which the majority of egg albumin was ovalbumin. In egg white, ovalbumin was the most abundant protein $[2,16]$.

Egg white albumin, which was already in the freeze dryer (CHRIST, ALPHA 1-2 LD Plus, Germany) at a temperature of $-70^{\circ} \mathrm{C}$, complexes with aspirin and caffeine. Aspirin, caffeine (SIGMA-Aldrich Co. LLC, China), and complex compounds were observed by electron spin resonance (ESR) to determine the ability as free radical scavenging, and by Fourier transform-infrared (FTIR) to determine the functional groups. The measurement conditions of ESR spectrometer (JEOL, JES-RE1X, Japan) were as follows: Center field: $335.6 \mathrm{mT}$; sweep width: $15 \mathrm{mT}$; and field modulation width: $0.5 \times 1 \mathrm{mT}$. To confirm functional groups of aspirin, caffeine, albumin, and their complex, FTIR spectrophotometer (SHIMADZU, 8400S, Japan) was set in the range of $400-4000 / \mathrm{cm}$ wave number. The ESR standard as free radical used 2.2-diphenyl-1-picrylhydrazyl (DPPH) compound (Fluka AG Buchs SG, Switzerland). This experiment also interested to know about the rheology of aspirin, egg white albumin, caffeine, and complex, which was called as viscosity. Viscosity measurement of individual component and complex scavenger was performed by a viscometer (DV-E Viscometer, BROOKFIELD, USA).

\section{RESULTS}

The results of docking of aspirin-ovalbumin-caffeine complex showed that aspirin and caffeine occurred at different docking site in the ovalbumin. Complex compounds formed by the hydrogen bond and hydrophobic interaction (Fig. 1a-c). The variability of tridimensional structures of aspirin and caffeine after binding to ovalbumin is shown in Fig. 1d-f.

The RMSD analysis showed that aspirin, as well as caffeine, altered the dynamic conformation of ovalbumin (Fig. 2). ESR results of DPPH, aspirin, egg white albumin, caffeine, and complex are shown in Fig. 3. FTIR spectra of aspirin, caffeine, egg white albumin, and its complex are shown in Fig. 4. Viscosities of egg white albumin, egg white albuminaspirin, egg white albumin-caffeine, and complex were 7.95, 5.53, 7.73, and 6.87 centipoises (cP), respectively.

\section{DISCUSSION}

\section{In silico analysis}

Interaction of aspirin-ovalbumin and caffeine-ovalbumin occurred at the different site of ovalbumin. This finding indicated that there was non-competitive docking site between aspirin and caffeine (Fig. 1a). It means that aspirin and caffeine attached at ovalbumin at separated area or they did not accumulate in the same site. Ovalbumin was

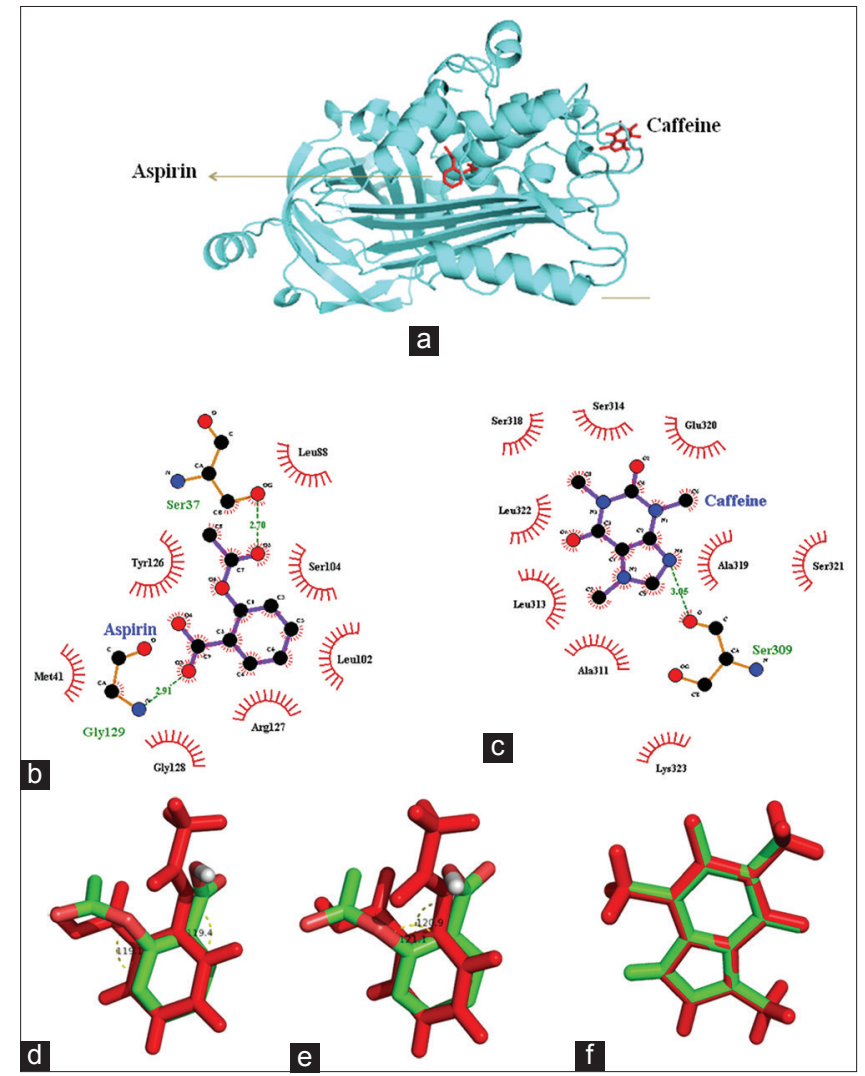

Fig. 1: Computational modeling. (a) The results of docking of aspirin-ovalbumin-caffeine. (b), (c) Hydrogen bonding which was shown with a green line and marked with a small line on the atom showed a hydrophobic bond. The shape of aspirin and caffeine structure, before (red) and after (green) docking (d), (e) Aspirin, and (f) Caffeine

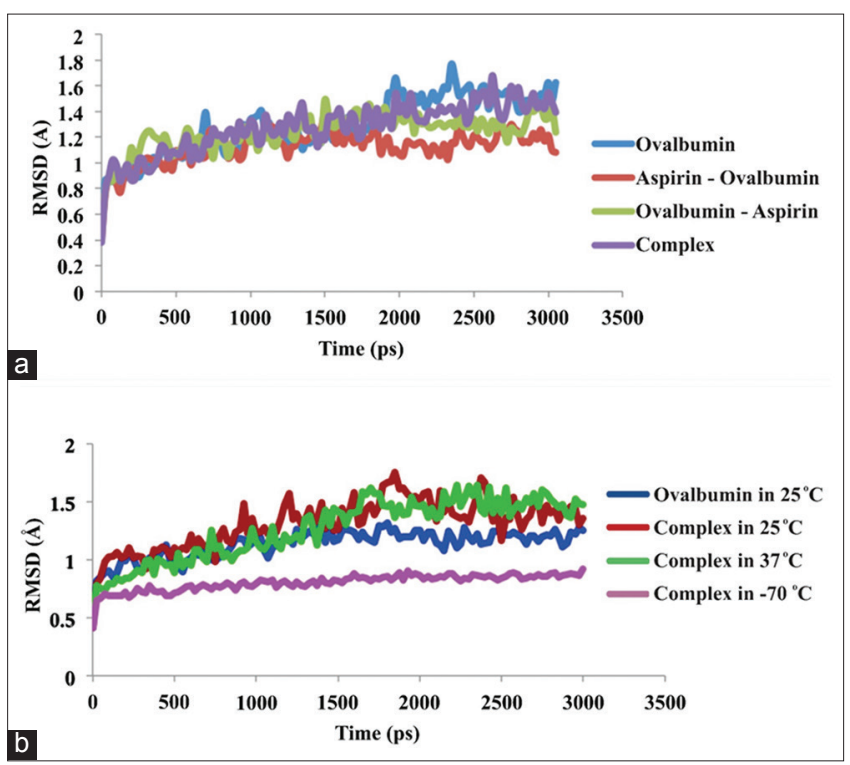

Fig. 2: The Root-Mean-Square-Deviation (RMSD) of ovalbumin, aspirin, and caffeine. (a) Comparison of single compounds and complex. (b) Temperature treatment, ovalbumin in a temperature of $25^{\circ} \mathrm{C}$ (blue line), complex in $25^{\circ} \mathrm{C}$ (red line), complex in $37^{\circ} \mathrm{C}$ (green line), and complex in $-70^{\circ} \mathrm{C}$ (purple line)

similar to albumin. Ovalbumin had non-specific binding properties to any compound, and they had transporter function. Ovalbumin 


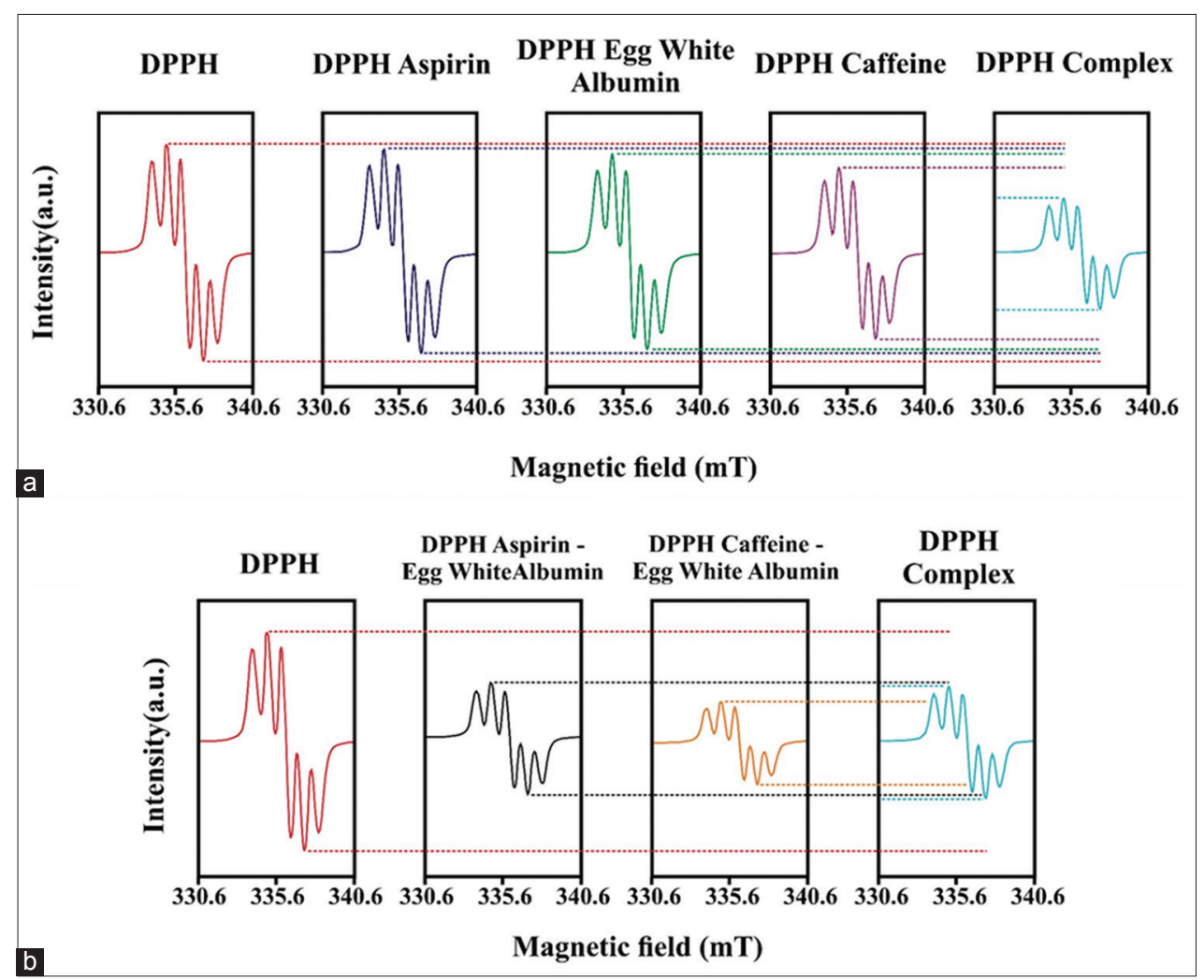

Fig. 3: ESR profiles of DPPH after it was added with aspirin, caffeine, egg white albumin, and complex (a), and DPPH after it was added with aspirin, egg white albumin, aspirin-egg white albumin, caffeine-egg white albumin, and complex (b)

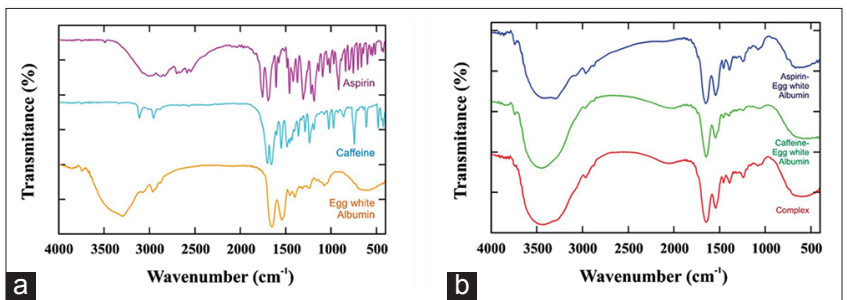

Fig. 4: FTIR spectra of aspirin, caffeine, and egg white albumin (a), and aspirin combined with egg white albumin, caffeine combined with egg white albumin, and complex (b)

had non-specific domain of aspirin and caffeine. Aspirin and caffeine bind to both hydrophilic and hydrophobic amino acids in ovalbumin. However, aspirin was slightly soluble with water, and it more likely binds to the hydrophobic amino acid. On the other hand, caffeine was more soluble with water. It bound to hydrophilic amino acid in ovalbumin. The successful docking, as illustrated in Fig. 1b and c, was indicated by the existence of hydrogen and hydrophobic bond between docking molecules (aspirin and caffeine) and amino acids of ovalbumin. Moreover, hydrogen bonds contributed the free energies of biological macromolecules and macromolecular complexes. It was a quantum mechanical phenomenon where hydrogen atoms were provided by covalent effects, polarization, and charge transfer [17].

Aspirin and caffeine bind to both hydrophilic and hydrophobic in amino acids of ovalbumin. Hydrophobic amino acids consisted of alanine (Ala), leucine (Leu), methionine (Met), and tyrosine (Tyr), whereas hydrophilic amino acids were composed by lysine (Lys), arginine (Arg), serine (Ser), and glutamine (Glu). Glycine was a particular amino acid that could act as both hydrophobic and hydrophilic [18].

The success of docking process showed that ovalbumin could serve as a transporter for both aspirin and caffeine. Albumin was also able to bind other molecules such as metals ions, fatty acids, drugs, and hormone. Such phenomenon above played an important role on the regulation of blood rheology of osmotic pressure, and on the distribution of fluid among different compartments, which was influenced by albumin. The flexible structure of albumin could adapt easily to ligands, whereas its domain design provided a variety of binding sites [19] and contributed easiness to receive and to release the ligand [3].

The variability of tridimensional structures of aspirin was shown after binding to ovalbumin. Aspirin model was rotated, and it resulted in the change of its angle. The angle of aspirin occurred on the base of carboxylic acid functional groups (Fig. 1d) were $119.1^{\circ}$ before the docking, and it became $119.4^{\circ}$ after the docking. Ester functional group (Fig. 1e) showed the changes in the angle of $120.9^{\circ}$ before the docking, and it became $121.1^{\circ}$ after the docking. This variability, however, could not be seen clearly on caffeine (Fig. 1f). Aliphatic compounds tended to accept several binding mechanisms because they were more flexible, for example, rotation, folding, etc. In contrast, an aromatic compound was less flexible because of rigid molecule. Both aspirin and caffeine contained the cyclic ring, but aspirin had another functional group (aliphatic chain), which was more flexible than caffeine. The angular changes that occurred in aspirin were caused by the atom rearrangements.

RMSD analysis showed that aspirin, as well as caffeine, altered the dynamic conformation of ovalbumin (Fig. 2a). Accordingly, ovalbumin, which was known to have the capacity to act as peptide antioxidant, showed cooperative and synergistically works of the amino acids when they conduct conformational changes [20]. Complex of RMSD (purple line) was almost coincident with ovalbumin line (blue line) and showed no significant chemical bond change to ovalbumin structure. This result meant that the nature of ovalbumin remained unchanged. Accordingly, this molecule was hard to be absorbed in the digestive system because intestinal enzymes degraded ovalbumin poorly [21]. Ovalbumin had a proteolytic digestion rate (even by pepsin) which was considerably slower than the rate of bovine hemoglobin or human 
globin hydrolysis [3]. This was the reason why ovalbumin did not affect in pharmacokinetics; ovalbumin also acted as a transporter of aspirin and caffeine. Furthermore, this finding that ovalbumin could be applied as an effective antioxidant peptide scavenger.

The complex of aspirin-ovalbumin-caffeine was treated at various temperatures such as a temperature of $25^{\circ} \mathrm{C}$ (room temperature), $37^{\circ} \mathrm{C}$ (body temperature), and $-70^{\circ} \mathrm{C}$ (freeze-drying temperature). RMSD of the complex was at $25^{\circ} \mathrm{C}$ (red line) and $37^{\circ} \mathrm{C}$ (green line) almost gave a same trend. However, a complex structure at a temperature of $-70^{\circ} \mathrm{C}$ (purple line) showed a similar RMSD trend like that of the ovalbumin (blue line). The complex of aspirin-ovalbumin-caffeine was treated at $-70^{\circ} \mathrm{C}$ showed intermolecular force (Fig. 2b). During freezedrying process, the complex had lost water to become a solid. Water loss affected to the chemical bond, in which, the random motion of molecules in the liquid state into vibration within molecules (stretching and bending). Accordingly, intermolecular forces became stronger which increased the magnetic force or the overall magnetic moment in the system because of the decreasing tendency toward the antiparallel coupling.

From the explanation above, we can conclude that magnetic field became stronger during the complex freezing. The motion of atoms in the complex was stable but not functional, which resulted in the strengthening of a magnetic bond while hydrophobic bond in the complex weakened. All atoms started sticking to each other. Then, it had a very regular pattern with molecules, which was rigidly close to each other that formed a crystalline lattice. Accordingly, the stability of complex structure increased (no denaturation) which could transform its function to be a stronger scavenger.

\section{ESR analysis}

Several methods were utilized for the determination of the radical scavenging effect of antioxidant molecules. The favored method was the DPPH method due to its simplicity with no requirement for a special tool and reaction. In addition, this method provided reliable and fast measurement. DPPH was a stable colored synthetic free radical which did not deteriorate in water and alcohols. The free radical scavenging activities of molecules depended on their ability to release hydrogen and to transform the structural conformation of their components [22,23]. Soares et al. [24] reported that DPPH easily accepted hydrogen or an electron from antioxidant molecules to convert into a stable diamagnetic molecule. In another report, Molyneux [25] stated that DPPH loss of violet color when it was mixed with a hydrogen donor substance. Discoloration occurred because of the decreasing quantity of DPPH radicals in the system. Therefore, the discoloration of the DPPH corresponded to the radical scavenging activity of the analyzed molecules.

In this study, the radical scavenging activities of aspirin, caffeine, egg white albumin, aspirin-egg white albumin, caffeine-egg white albumin, and aspirin-egg white albumin-caffeine (complex) were studied using ESR with DPPH as the free radical. As shown in Fig. 3, DPPH had the highest peak. The DPPH peak decreased more drastically when the complex compound (aspirin-egg white albumin-caffeine) was added, rather than the addition of aspirin, egg white albumin, and caffeine (Fig. 3a). Reduction in the peak reflected a decline in the quantity of the DPPH as free radical. The complex compound had plenty of electrons that could be donated to the DPPH through electron mobile or transition. Then, the resulted electron pairs caused an intensity decreasing of the DPPH as free radical. In addition, to donate electrons, the complex compounds could also accept excess electrons from an atom so that the formation of a new radical can be avoided. It meant that the complex compound could act as the comprehensive free radical scavenger. When aspirin or caffeine was used as a single antioxidant, it was possible that it could generate the existence of a new radical.

The free radical chain reaction could be interrupted by the presence of antioxidants. Antioxidants donated $\mathrm{H} \bullet$ to the free radical, which was formed during oxidation of the radical itself, which usually contained aromatic or phenolic compounds [26]. When a combination of the aspirin-egg white albumin or the caffeine-egg white albumin was added, the peak of the DPPH radical decreased lower than the single addition of aspirin and caffeine. Moreover, the addition of the caffeineegg white albumin to the DPPH resulted in lower peak intensity than the addition of the complex compound (Fig. 3b). Although it was an interesting phenomenon, this mechanism was still not clear. Thus, it needs further investigations.

Several studies using a single antioxidant have been conducted using aspirin [27], indomethacin [27], and astaxanthin [28]. Although these compounds could inhibit spin adduct formation, in the presence of relatively high concentration of reactive oxygen species, the formation of new radical species cannot be avoided. Such results indicated that our study by employing the complex antioxidant is more effective than a single antioxidant due to the existence of high amount of electron in the complex. If there is excess of reactive oxygen species, its effect would be reduced during the cycling redox process.

\section{FTIR analysis}

The FTIR analysis of aspirin, caffeine, and egg white albumin is shown in Fig. 4a. The sharp peaks of aspirin were at 1693.38, 1188.07, and $916.12 / \mathrm{cm}$. It correlated with the stretching of $\mathrm{C}=0$ bond (carboxylic acids), the waging of $\mathrm{C}-\mathrm{H}$ bond (alkyl halides), and the bending of $\mathrm{O}-\mathrm{H}$ bond (carboxylic acids). The sharp peaks of caffeine were at 1656.74 and $742.54 / \mathrm{cm}$. It correlated with the stretching of the $\mathrm{C}=\mathrm{C}$ bond (alkenes) and $\mathrm{C}-\mathrm{H}$ bond (alkenes). Aspirin and caffeine had an aromatic ring. However, they were not at a sharp peak (aspirin: 1575.73 and caffeine: 1598.88/cm). Albumins have three sharp peaks as follows: 3296.12 , 1650.95 , and $1541.02 / \mathrm{cm}$. It correlated with the stretching of $\mathrm{O}-\mathrm{H}$ bond (carboxylic acid), the stretching of the $\mathrm{C}=\mathrm{C}$ bond (alkenes), and an aromatic ring. The FTIR analysis of the aspirin-albumin, the caffeinealbumin, and the complex compound (aspirin-albumin-caffeine) is given in Fig. 4b. The sharp peaks of aspirin-albumin were at 3296.12, 1649.02 , and $698.18 / \mathrm{cm}$. It correlated with the stretching of O-H bond ( $\mathrm{H}$-bonded), $\mathrm{C}=\mathrm{O}$ bond, and the bending of $\mathrm{C}-\mathrm{H}$ bond (aromatic).

The sharp peaks of caffeine-albumin were at $3448.49,1647.10$, and $1544.88 / \mathrm{cm}$. It correlated with the stretching of $\mathrm{O}-\mathrm{H}$ bond (H-bonded), $\mathrm{C}=\mathrm{O}$ bond, and $\mathrm{C}=\mathrm{C}$ bond (aromatic). The complex compound had several peaks at $3450.41,1649.02,1542.95$, and $665.40 / \mathrm{cm}$. It correlated with the stretching of $\mathrm{O}-\mathrm{H}$ bond (H-bonded), the stretching of $\mathrm{C}=\mathrm{O}$ bond (Amide bond), the bending of $\mathrm{C}=\mathrm{C}$ bond (aromatic), and the waging of $\mathrm{N}-\mathrm{H}$ bond (1.2 amines). FTIR analysis of aspirin-egg white albumin resulted in a chemical reaction producing $\mathrm{C}-\mathrm{N}$ or $\mathrm{C}=0$ groups at a a wavenumber of 1649.02 . On the other hand, caffeine-egg white albumin which was the amino group in egg white albumin reacted on the $\mathrm{C}=0$ group with caffeine to $\mathrm{C}-\mathrm{O}$ or $\mathrm{C}-\mathrm{N}$ groups, which was detected at a wavenumber of 1074.28. The FTIR analysis results showed a chemical interaction between the complex of aspirin-egg white albumin-caffeine, which were shown by the stretching of $\mathrm{C}=0$ bond at a wavenumber of 1649.02 that was the amide. All FTIR analyses in this study showed the existence of aromatic groups, which were useful for the scavenger molecules.

\section{Viscosity analysis}

Egg white albumin (as a control) had a viscosity of $7.95 \mathrm{cP}$. When egg white albumin was added into aspirin, its viscosity decreased to $5.53 \mathrm{cP}$. Aspirin caused the phenomena of dilution, which was showed by the decreasing viscosity. The phenomena are similar to human serum blood thinning when interacting with aspirin. Gibb energy distribution changed along with conformational dynamics of the complex of albumin-aspirin [29]. On the other hand, caffeine did not significantly change the viscosity; the viscosity was found to be $7.73 \mathrm{cP}$, which was close to egg white albumin itself. The viscosity of the complex (aspirinegg white albumin-caffeine) was $6.87 \mathrm{cP}$, which was almost similar to the viscosity of stomach, as well as duodenal mucin [30]. It indicated that the complex of aspirin-egg white albumin-caffeine was a proper 
viscosity for a therapeutic agent when it was applied for antioxidants scavenger in the digestive tract system. The complex of ovalbumin had similar viscosity with normal gastric physiology, with the same value of normal duodenal human mucus, which was $6.1 \mathrm{cP}$ [31]. Mucus was capable to act as an antioxidant and to reduce mucosal damage mediated by oxygen free radicals [32]. Mucus gel had several functions, it acted as lubrication for the passage of objects, maintenance of hydrated layer over the epithelium, a permeable gel layer for the exchange of gases and nutrients with the underlying epithelium, and a barrier to pathogens, noxious substances, nutrients, and enteric drugs that must interact and diffuse [33].

\section{CONCLUSION}

The characters of albumin when it binds to aspirin and caffeine indicated that scavenging activity of the complex and the viscosity showed an important to be physiological scavengers of free radicals. Both aspirin and caffeine were not competitively bind to albumin. They also have a non-specific binding location to albumin.

\section{ACKNOWLEDGMENTS}

All authors would like to thank Research Institute of Free Radical Shedding Malang, Prof. Widodo, S.Si, MSi, Ph.D. Med.Sc., Didik Huswo Utomo, M.Si., and Rizky Arief Sobirin, M.Si. The authors specifically thank Research Institute of Free Radical Shedding, Malang, Indonesia, for funding this research.

\section{AUTHOR'S CONTRIBUTIONS}

All authors have their respective contributions to this journal, namely Sutiman Bambang Sumitro: Conducting ESR analysis and editing the manuscript, Gatra Ervi Jayanti: Conducting in silico analysis and writing the draft of the journal, Akhmad Sabarudin: Conducting FTIR analysis and editing the manuscript, and Sri Widyarti: Conducting viscosity analysis and editing the manuscript.

\section{CONFLICTS OF INTEREST STATEMENT}

The authors have declared no conflicts of interest

\section{REFERENCES}

1. Davalos A, Miguel M, Bartolome B, Lopez-Fandino R. Antioxidant activity of peptides derived from egg white proteins by enzymatic hydrolysis. J Food Prot 2004;67:1939-44.

2. Kovacs-Nolan J, Phillips M, Mine Y. Advances in the value of eggs and egg components for human health. J Agric Food Chem 2005;53:8421-31.

3. Walker V, Taylor WH. Ovalbumin digestion by human pepsins 1, 3 and 5. Biochem J 1978;176:429-32.

4. Nyemb K, Jardin J, Causeur D, Guerin-Dubiard C, Dupont D, Rutherfurd SM, et al. Investigating the impact of ovalbumin aggregate morphology on in vitro ovalbumin digestion using label-free quantitative peptidomics and multivariate date analysis. Food Res Int 2014;63:192-202.

5. Baynes JW, Dominiczak MH. Medical Biochemistry. $2^{\text {nd }}$ ed. Maryland (USA): Mosby Inc; 2004.

6. Guerrero A, Gonzalez-Correa JA, Arrebola MM, Munoz-Marin J, De La Cuesta FS, De La Cruz JP. Antioxidant effects of a single dose of acetylsalicylic acid and salicylic acid in rat brain slices subjected to oxygen-glucose deprivation in relation with it's antiplatelet effect. J Neulet 2004;358:153-56.

7. Khan MF, Bin Rashid R, Rahman MM, Al Faruk MD, Rahman MD, Rashid MA. Effects of solvent polarity on solvation free energy, dipole moment, polarizability, hyperpolarizability and molecular reactivity of aspirin. Int J Pharm Pharm Sci 2017;9:217-21.

8. Agyemang-Yeboah F, Oppong SY. Caffeine: The wonder compound, chemistry, and properties. Top Ser Health Sci 2013;1:27-37.
9. Shah KM, Dadhaniya P, Trivedi VR. Effect of caffeine in experimental model of rheumatoid arthritis in rats. Int $\mathrm{J}$ Pharm Pharm Sci 2015;7:365-7.

10. NCBI. Ovalbumin. Available from: https://www.ncbi.nlm.nih.gov 2006.

11. PubChem. Aspirin. Caffeine. Available from: https://www.pubchem. ncbi.nlm.nih.gov/2006

12. Trott $\mathrm{O}$, Olson AJ. AutoDock Vina: Improving the speed and accuracy of docking with a new scoring function, efficient optimization, and multithreading. J Comput Chem 2010;31:455-61.

13. Pymol. The PyMol Molecular Graphics System. Version 1.3. Scrodinger: LLC; 2010.

14. Wallace AC, Laskowski RA, Thornton JM. LIGPLOT: A program to generate schematic diagrams of protein-ligand interaction. Protein Eng 1995;8:127-34.

15. Krieger E, Darden T, Nabuurs SB, Finkeistein A, Vriend G. Making optimal of empirical energy functions: Force-field parameterization in crystal space. Proteins Struct Funct Bioinf 2004;57:678-83.

16. Nisbet AD, Saundry RH, Moir AJ, Fothergill LA, Fothergill JE. The complete amino-acid sequence of hen ovalbumin. Eur J Biochem 1981;115:335-45.

17. Morozov AV, Kortemme T. Potential functions for hydrogen bonds in protein structure prediction and design. Adv Protein Chem 2005;72:1-38

18. Lodish H, Berk A, Matsudaira P, Kaiser CA, Krieger M, Scott MP, et al. Molecular Cell Biology. $5^{\text {th }}$ ed. Madison Avenue (NY): WH Freeman and Company; 2004.

19. Roche M, Rondeau P, Singh NR, Tarnus E, Bourdon E. Edited by barry halliwell. the antioxidant properties of serum albumin. FEBS Lett 2008;582:1783-87

20. Guo F, Li SC, Ma W, Wang L. Detecting protein conformational changes in interactions via scaling known structures. J Comput Biol 2013;20: 765-79.

21. Oliveira FM, Dos Santos EM, Alves AC, Campana-Pereira MA, Ramaldes GA, Cardoso VN, et al. Digestion, absorption and tissue distribution of ovalbumin and palmitoyl-ovalbumin: Impact on immune responses triggered by orally administered antigens. Scand J Immunol 2007;65:139-47.

22. Fukumoto LR, Mazza G. Assessing antioxidant and prooxidant activities of phenolic compounds. J Agric Food Chem 2000;48:3597-604.

23. Shimada K, Fujikawa K, Yahara K, Nakamura T. Antioxidative properties of xanthone on the auto oxidation of soybean in cylcodextrin emulsion. J Agric Food Chem 1992;40:945-8

24. Soares JR, Dinis TC, Cunha AP, Almeida LM. Antioxidant activity of some extracts of Thymus zygis. Free Radic Res 1997;26:469-78.

25. Molyneux P. The use of the stable free radical diphenylpicrylhydrazyl (DPPH) for estimating antioxidant activity. Songklanakarin J Sci Technol 2004;26:211-9.

26. Brewer MS. Natural antioxidants: Sources, compounds, mechanisms of action, and potential applications. Compr Rev Food Sci Food Saf 2011;10:221-47.

27. Turnbull CM, McClure D, Rossi AG, Megson IL. A novel electron paramagnetic resonance-based assay for prostaglandin $\mathrm{H}$ synthase-I activity. J Inflamm 2006;3:12.

28. Polyakov NE, Magyar A, Kispert LD. Photochemical and optical properties of water-soluble xanthophyll antioxidants: Aggregation vs complexation. J Phys Chem B 2013;117:10173-82.

29. Alvarez HA, Mc Carthy AN, Grigera JR. A molecular dynamics approach to ligand-receptor interaction in the aspirin-human serum albumin complex. J Biophys 2012;2012:642745.

30. Georgiades P, Pudney PD, Thornton DJ, Waigh TA. Particle tracking microrheology of purified gastrointestinal mucins. Biopolymers 2014;101:366-77.

31. Curt JR, Pringle R. Viscosity of gastric mucus in duodenal ulceration. Gut 1969;10:931-4.

32. Repetto MG, Llesuy SF. Antioxidant properties of natural compounds used in popular medicine for gastric ulcers. Braz J Med Biol Res 2002;35:523-34.

33. Bansil R, Turner BS. Mucin strycture, aggregation, physiological functions and biomedical applications. Curr Opin Colloid Interface Sci 2006; $11: 164-70$. 